\title{
A remark on homomorphisms between generalized Verma modules
}

\author{
By
}

\section{Akihiko GYOJA}

1. Let $g$ be a complex simple Lie algebra, $\mathfrak{p}_{s}$ a parabolic subalgebra, and $\hat{\mathfrak{p}}_{s}$ the set of (isomorphism classes of) simple $\mathfrak{p}_{s}$-modules of finite dimension. For $E \in \hat{p}_{s}$, put $M_{s}(E)=U(\mathfrak{g}) \otimes_{U\left(\mathfrak{p}_{0}\right)} E$, where $U(-)$ denotes the enveloping algebra.

In many cases, it is known that

$$
\operatorname{dim} \operatorname{Hom}_{8}\left(M_{s}(E), M_{s}(F)\right) \leq 1 \text { for } E, F \in \hat{p}_{s}
$$

$([7],[1], \cdots)$. Based on these results, it was once conjectured that $(\mathrm{A})$ is always valid. But R.S.Irving $[3,9.6]$ produced an example that

$$
\operatorname{dim} \operatorname{Hom}_{8}\left(M_{s}(E), M_{s}(F)\right)=2, \mathrm{~g}=D_{4},
$$

and this conjecture has been negatively settled. See also [4].

Recently, the author encountered a curious phenomenon. Generalizing [6], we can show a relation between

(1) irreducible factors of $b$-functions of semi-invariants, and

(2) intertwining operators between generalized Verma medules.

Thus it would be natural to expect that the dimensions of these Hom spaces should be related with the multiplicities of the irreducible factors of the $b$-functions. Curiously, it turned out that the dimension of these Hom spaces do not play any role concerning this point. To explain this phenomenon, arises the following question.

(B) Is $\operatorname{Hom}_{8}\left(M_{s}(E), M_{s}(F)\right)$ irreducible in some sense?

In this note, we take up example of Irving [3] and give an affirmative answer to (B) in this case.

2. Fix a Cartan subalgebra $\mathfrak{h}$ of a complex simple Lie algebra $\mathfrak{g}$, a root basis $\left\{\alpha_{i}\right\}$, and a root vector $X_{i}$ for each $\alpha_{i}$. Take the root vector $Y_{i}$ for the root $-\alpha_{i}$ so that $\alpha_{i}\left(\left[X_{i}, Y_{i}\right]\right)=2$. Let $\Gamma$ be the stabilizer of $\left(\mathfrak{h},\left\{\alpha_{i}\right\},\left\{X_{i}\right\}\right)$ in Aut $(\mathrm{g})$. Then Aut $(\mathrm{g})$ is a semidirect product of $\Gamma$ and the inner automorphism 
group of g. Let $S$ be a $\Gamma$-stable subset of $\left\{\alpha_{i}\right\}$, pps the corresponding parabolic subalgebra, and $\hat{p}_{s, \Gamma}$ the set of $E \in \hat{p}_{s}$ whose highest weight is $\Gamma$-invariant.

Lemma. Any $E \in \hat{p}_{s, \Gamma}$ has a unique $\Gamma$-module structure such that a highest weight vector is $\Gamma$-invariant and $\gamma(u e)=\gamma(u) \gamma e\left(\gamma \in \Gamma, u \in U\left(\mathfrak{p}_{s}\right), e \in E\right)$.

Proof. Let $e_{0}$ be a highest weight vector. It suffices to prove that $\gamma(u) e_{0}$ $\left(\gamma \in \Gamma, u \in U\left(\mathfrak{p}_{s}\right)\right)$ depends only on $u e_{0}$, or equivalently, that the annihilator of $e_{0}$ in $U\left(\mathfrak{p}_{s}\right)$ is $\Gamma$-stable. The latter can be proved using the explicit description of the annihilator [2, 7.2.7(iii)].

For $E \in \hat{p}_{s, \Gamma}$, define the $\Gamma$-module structure of $M_{s}(E)$ by $\gamma(u \otimes e)=$ $\gamma(u) \otimes \gamma e(\gamma \in \Gamma, u \in U(\mathrm{~g}), e \in E)$. For $E, F \in \mathfrak{p}_{s, \Gamma}$, define the $\Gamma$-module structure of $H:=\operatorname{Homg}\left(M_{s}(E), M_{s}(F)\right)$ by $\gamma(h)(m)=\gamma\left(h\left(\gamma^{-1} m\right)\right)(\gamma \in \Gamma, h \in H, m$ $\in M_{s}(E)$ ).

3. Let $\mathrm{g}$ be the complex simple Lie algebra of type $D_{4},\left\{\alpha_{i}\right\}_{0 \leq i \leq 3}$ be the simple roots where $\alpha_{0}$ is $\Gamma$-invariant, $\left\{\pi_{i}\right\}$ the fundamental weights, and $\left\{s_{i}\right\}$ the simple reflections. Put $x=s_{0}, y=s_{0} s_{1} s_{2} s_{3} s_{0}, \lambda_{n}=x \cdot\left(-n \pi_{0}-\rho\right)$, and $\mu_{n}=y \cdot\left(-n \pi_{0}\right.$ $-\rho)(n=1,2, \cdots)$. Here $w \cdot \nu=w(\nu+\rho)-\rho$ with $\rho=\sum \pi_{i}$. (Note that $\lambda_{n}=(n$ $-1) \pi_{0}-(n+1)\left(\pi_{1}+\pi_{2}+\pi_{3}\right)$ and $\mu_{n}=(2 n-1) \pi_{0}-(n+1)\left(\pi_{1}+\pi_{2}+\pi_{3}\right)$.) Let $S=$ $\left\{\alpha_{0}\right\}, E_{n}$ (resp. $F_{n}$ ) be the simple $\mathfrak{p}_{s}$-module whose highest weight is $\lambda_{n}$ (resp. $\left.\mu_{n}\right)$, and $e_{n}$ (resp. $f_{n}$ ) its highest weight vector. In the present case, $\Gamma \simeq \widetilde{S}_{3}$. Take an element $\delta \in \Gamma$ of order 3 , put $\omega:=\exp (2 \pi \sqrt{-1} / 3)$, and

$$
u_{1}:=\sum_{i=0}^{2} \omega^{i} \delta^{-i}\left(Y_{10203}-2 Y_{10230}-Y_{12030}\right)
$$

where $Y_{i j k l m}:=Y_{i} Y_{j} Y_{k} Y_{l} Y_{m}$. Using the relations $Y_{0}^{2} f_{1}=0,\left[Y_{0},\left[Y_{0}, Y_{1}\right]\right]=0$, $\left[\left[Y_{0}, Y_{1}\right],\left[Y_{0}, Y_{2}\right]\right]=0$ and their $\Gamma$-conjugates, we can show that $u_{1} \otimes f_{1}$ is of weight $\lambda_{1}$, is annihilated by $\left\{X_{i}\right\}, \delta\left(u_{1} \otimes f_{1}\right)=\omega\left(u_{1} \otimes f_{1}\right)$, and the weight space $M_{s}\left(F_{1}\right)^{\lambda_{1}}$ is spanned by the 14 elements $Y_{i j k l m} \otimes f_{1}$ with $(i j k l m) \in\{(01023)$, (01203), (02301), (03102), (01230), (10203), (20301), (30102), (10230), (20310), (30120), (12030), (23010), (31020)\}. Since $\operatorname{dim} M_{s}\left(F_{1}\right)^{\lambda_{1}}=14$, these elements are linearly independent, and hence $u_{1} \otimes f_{1} \neq 0$. Define a homomorphism $h_{1}$ : $M_{S}\left(E_{1}\right) \rightarrow M_{S}\left(F_{1}\right)$ so that $h_{1}\left(1 \otimes e_{1}\right)=u_{1} \otimes f_{1}$. Then $h_{1} \neq 0$ and $\delta\left(h_{1}\right)=\omega h_{1}$. By [5, 1.17], $M_{s}\left(E_{1}\right)$ is simple, and hence $h_{1}$ is injective. By [5, 2.11 and 2.25], we get

$$
M_{s}\left(E_{n}\right) \simeq T_{-\pi_{0}-\rho}^{-n \pi_{0}-\rho} M_{S}\left(E_{1}\right)=\left(M_{S}\left(E_{1}\right) \otimes V\left(-(n-1) \pi_{0}\right)\right)_{-n \pi_{0}-\rho}
$$

and a similar relation for $F_{n}$. (See [5] for the definitions of $T_{\lambda^{\prime}}^{\lambda^{\prime}}, \mathrm{V}\left(\lambda^{\prime}-\lambda\right)$, and ()$\left._{\lambda^{\prime} .}\right)$ Then $h_{1} \otimes\left(\right.$ identity) induces an injective homomorphism $h_{n} \in \mathrm{Hom}_{8}$ $\left(M_{s}\left(E_{n}\right), M_{s}\left(F_{n}\right)\right)=: H_{n}$ such that

$$
\delta\left(h_{n}\right)=\omega h_{n} .
$$

Here we have applied the lemma in $\S 2$ to $V\left(-(n-1) \pi_{0}\right)$. Since $\operatorname{dim} H_{n}=2$ 
[3, 9.6], the existence of such $h_{n} \in H_{n} \backslash\{0\}$ implies the irreducibility of the $\Gamma$-module $H_{n}$.

4. Define $h_{n}^{\prime} \in H_{n}$ in the same way as $h_{n}$ using $\omega^{-1}$ instead of $\omega$. Then $H_{n}=C h_{n} \oplus C h_{n}^{\prime}$. Put $\quad N:=M_{s}\left(E_{1}\right) \otimes V\left(-(n-1) \pi_{0}\right)$, and $N^{\prime}:=$ $M_{s}\left(F_{1}\right) \otimes V\left(-(n-1) \pi_{0}\right)$. Then $h \rightarrow h \otimes\left(\right.$ identity) gives an injection $H_{1} \rightarrow$ Hom $_{8}$ $\left(N, N^{\prime}\right)$. Decomposing $N=\oplus_{\nu} N_{\nu}$ and $N^{\prime}=\oplus_{\nu} N_{\nu}^{\prime}$ according to the central character, we get a projection $\operatorname{Hom}_{8}\left(N, N^{\prime}\right) \rightarrow H_{n}$. Composing these mappings, we get a linear isomorphism $\varphi: H_{1} \rightarrow H_{n}$ such that $\varphi\left(h_{1}\right)=h_{n}$ and $\varphi\left(h_{1}^{\prime}\right)=h_{n}^{\prime}$. Calculating the $\Gamma$-action on $h_{1}, h_{1}^{\prime}, h_{n}$ and $h_{n}^{\prime}$, we can show that $\varphi$ is $\Gamma$ equivariant. In other words, the $\Gamma$-action on $H_{n}$ can be also defined from the $\Gamma$-action on $H_{1}$, identifying $H_{1}$ and $H_{n}$ by $\varphi$. Thus we can define the $\Gamma$-action on $H_{n}$ without applying the lemma in $\S 2$ to $V\left(-(n-1) \pi_{0}\right)$. This remark enables us to apply the argument in $\S 3$ to the example of Irving-Shelton [4, 5.3], which includes the example of Irving [3, 9.6] as a special case.

5. Let us consider the general case. As for the candidates for the group acting on the Hom space, the outer automorphism group of $\mathrm{g}$ is too small. In fact, starting from the example of Irving $[3,9.6]$, and using the transitivity of the induction [2, 5.1.11 and 5.1. 12], we get examples such that $\operatorname{dim}$ Hom $\geq 2$ and such that the Dynkin diagram of $g$ is connected and strictly larger than that of $D_{4}$. Then the outer automorphism group of $\mathrm{g}$ is $\boldsymbol{Z} / 2 \boldsymbol{Z}$ or $\{0\}$, which can not act on the Hom space whose dimension is $\geq 2$.

\section{DEPARTMENT OF FUNDAMENTAL SCIENCES, FACULTY OF INTEGRATED HUMAN STUDIES, KYOTO UNIVERSITY.}

\section{References}

[1] B. D. Boe, D. H. Collingwood, A comparison theorem for the structure of induced representations, J. Algebra, 94 (1985), 511-545.

[2] J. Dixmier, Algèbres enveloppantes, Gauthier-Villars, Paris, 1974.

[3] R. S. Irving, Projective modules in the category Os : self-duality, Trans. AMS, 291 (1985), 701-732.

[4] R. S. Irving, B. Shelton, Loewy series and simple projective modules in the category $\mathcal{O}$ s, Pacific J. Math., 132 (1988), 319-342.

[5] J. C. Jantzen, Moduln mit einem höchsten Gewicht, Lecture Notes in Math., 750 (1979), Springer.

[6] M. Kashiwara, The universal Verma module and the b-function, Advanced Studies in Pure Math., 6 (1985), 67-81.

[ 7 ] J. Lepowsky, Uniqueness of embeddings of certain induced modules, Proc. AMS, 56 (1976), 55 58. 